\title{
Lung Volume Recruitment in Lambs during High-Frequency Oscillatory Ventilation Using Respiratory Inductive Plethysmography
}

\author{
SYLVIA GÖTHBERG, THOMAS A. PARKER, JEFF GRIEBEL, \\ STEVEN H. ABMAN, AND JOHN P. KINSELLA \\ Department of Pediatric Anesthesia and Intensive Care, The Queen Silvia Children's Hospital, 41685 \\ Göteborg, Sweden [S.G.]; and Pediatric Heart Lung Center, Department of Pediatrics, Sections of \\ Neonatology [T.A.P., J.P.K.] and Pulmonary Medicine [J.G., S.H.A.], The Children's Hospital and the \\ University of Colorado School of Medicine, Denver, Colorado 80218, U.S.A.
}

\begin{abstract}
Monitoring lung volume is important in the treatment of acute hypoxemic respiratory failure. However, there are no tools available for lung volume measurement to guide ventilator management during high-frequency oscillatory ventilation (HFOV) and during dynamic changes in conventional ventilation (CV). We studied the performance of a new respiratory inductive plethysmograph (RIP) with modified software. We measured $\Delta$ changes in lung volume above end-expiratory volume $\left(\mathrm{V}_{\mathrm{RIP}}\right)$ during HFOV and studied whether changes in $V_{\text {RIP }}$ parallel changes in mean airway pressure. Calibration of the plethysmograph was made by serial injections of a known gas volume in six term (140 $\mathrm{d}$ gestation) and eight preterm (125 d gestation) lambs. Linear regression analysis of the relationship between injected gas volume and $\mathrm{V}_{\mathrm{RIP}}$ showed strong correlation $\left(r^{2}=0.93-1.00\right.$ term animals, $r^{2}=0.86-1.00$ preterm animals). The pressure volume curves from the calibration with the injected gas volumes also correlated well with the pressure volume curves extrapolated from changes in $\mathrm{V}_{\text {RIP. }}$. Lung hysteresis was clearly demonstrated with RIP after changes in mean airway pressure during HFOV and after changes in positive end-expiratory pressure during $\mathrm{CV}$. We conclude that measurements of lung volume in term and preterm lambs by use of modified RIP correlate well with changes in mean airway pressure during HFOV, with static pressure volume curves and with changes in positive endexpiratory pressure during $\mathrm{CV}$. We speculate that this technique may provide clinically useful information about changes in lung
\end{abstract}

\section{ABSTRACT}

volume during HFOV and CV. However, evaluation of the precision and chronic stability of RIP measurements over prolonged periods will require further studies. (Pediatr Res 49: $38-44,2001$ )

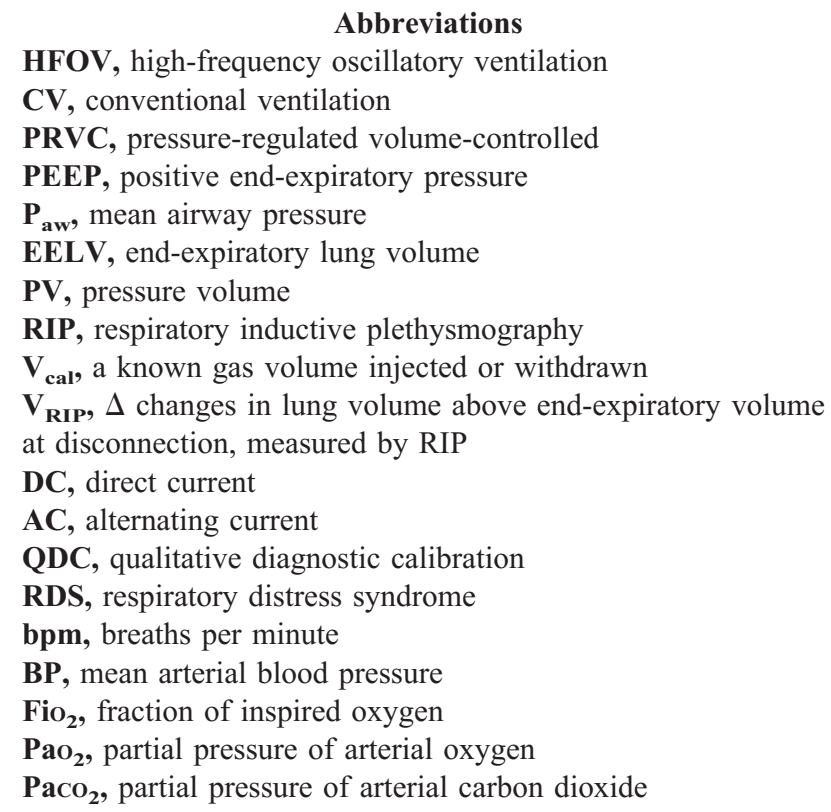

Monitoring lung volume is important in the treatment of hypoxemic respiratory failure, but few noninvasive tools are available to guide ventilator management. Numerous studies

Received May 24, 2000; accepted June 22, 2000

Correspondence: Sylvia Göthberg, M.D., Department of Anesthesia and Intensive Care, The Queen Silvia Children's Hospital, S-416 85 Göteborg, Sweden.

Supported by the Swedish Heart and Lung Foundation, Children's Hospital Research Foundation (Göteborg, Sweden), Göteborg's Society of Medicine (Sweden), The Medical Faculty of Göteborg (LUA), Newborn Hope Inc. (Colorado, U.S.A.); American Heart Association Established Investigator Award; and NIH HLBI 01942 and 57144. have demonstrated the importance of monitoring lung volume during mechanical ventilation to improve oxygenation (1-17). Minimizing volutrauma and lung injury is equally important (18-24). Targeting lung volume rather than gas exchange alone may improve survival in patients with acute hypoxemic respiratory failure $(25,26)$. A reliable noninvasive bedside technique to measure changes in lung volume in response to changes in $\mathrm{P}_{\mathrm{aw}}$ during HFOV would be an important tool for improving lung volume-targeted ventilation. Lung volume evaluation is important during initial alveolar recruitment and 
after disconnection and suctioning. Efforts have been made to measure lung volumes during HFOV. However, the methods used include interruption of HFOV for nitrogen washout on $\mathrm{CV}$ (27) or are too time consuming for measuring rapid dynamic changes (28). Monitoring dynamic changes in lung volume during $\mathrm{CV}$ could also be used to modify ventilator settings after changes in PEEP and after surfactant instillation.

RIP is a noninvasive technique for measuring changes in lung volume $(29,30)$. The method employs thoracic and abdominal elastic bands that register changes in inductance proportional to changes in thoracic and abdominal volume. RIP has been used to measure tidal volume (TV) and minute ventilation and correlates well with pneumotachography in infants and children (31-34). However, measurement of changes in lung volume by use of RIP has been limited by baseline drift and nonlinearity of the measurement at extremes of lung inflation (35).

Recently, software modifications that were designed to allow measurements of lung volume when used in DC-coupled mode were incorporated into a RIP system (RespiTrace, SensorMedics Corp., Yorba Linda, CA, U.S.A.). Our aim with the present study was to determine whether RIP could be used during HFOV and whether RIP measurements of lung volume would respond immediately to changes in $\mathrm{P}_{\mathrm{aw}}$ and oxygenation. We also studied the immediate changes in lung volume as measured by RIP after changes in PEEP during CV. We hypothesized that $\mathrm{V}_{\mathrm{RIP}}$ would correlate with changes in $\mathrm{V}_{\text {cal }}$. We also hypothesized that changes in $\mathrm{V}_{\mathrm{RIP}}$ would correlate with dynamic changes in lung volume during recruitment and derecruitment maneuvers on HFOV and during $\mathrm{CV}$ of term animals.

In addition to studies during mechanical ventilation of term newborn lambs, we also applied the RIP technique to preterm lambs with severe RDS. Mechanical ventilation of preterm lambs delivered at $125 \mathrm{~d}(0.85 \%$ gestation $)$ provides a natural model of severe RDS caused by surfactant deficiency. This model is characterized by progressive hypoxemia and lung injury during the first hour after delivery and mechanical ventilation $(36,37)$. In this model of severe RDS, our aim was to correlate $V_{\text {RIP }}$ to injections of known gas volumes before and after surfactant treatment. We hypothesized that PV curves extrapolated from RIP measurements would correlate with PV curves from the calibration with injected gas volumes and that these curves would demonstrate less hysteresis in preterm than in term animals. We also hypothesized that increased hysteresis after surfactant instillation could be demonstrated with RIP.

\section{METHODS}

The present study was reviewed and approved by the Animal Care and Use Committee at the University of Colorado Health Sciences Center. The handling of the animals was in accord with the guidelines of the National Institutes of Health.

\section{RIP: Technology}

RIP is a respiratory transducer that measures the crosssectional areas of the rib cage and abdomen from changes in electrical inductance. It is composed of two transducers of
Teflon-coated highly flexible and multistranded wires fastened in zigzag form to an elastic band. The zigzag nature of the wire forms the inductance coil for the oscillator electronics. Expansion and contraction of these coils alter the inductance that is converted into proportional voltages by use of a variable frequency oscillator connected to each coil. The signal is isolated with an optocoupler that provides isolation between the oscillator and the subject. The outputs from the optocouplers are sent to phase-locked loops that demodulate the signals and provide DC output signals. These signals are then fed through band-pass filters for the AC-coupled mode and lowpass filters for the DC-coupled mode. The AC-coupled mode has the ability to restore its output to a fixed baseline and allows gains, giving it the maximal dynamic range for TV breathing. The DC-coupled mode is used to reflect changes in resting lung volume $(29,30,38,39)$.

After placement of the RIP bands around the thorax and abdomen, the device is internally calibrated using a QDC procedure. QDC uses a statistical selection of breaths from a calibration period of $5 \mathrm{~min}$. Recent modifications in the software of this device allow assignment of a numerical value for mean lung volume when used in the DC-coupled mode. The RIP was used in DC-coupled mode and was connected to a computer with a serial cable. The measured changes in the electrical inductance were recorded from the computer screen during the study.

\section{Surgical Preparation}

Mixed-breed (Columbia-Rambouillet, Nebekar Ranch, Lancaster, CA, U.S.A.) pregnant ewes at either term, 138-147 d gestation (term $147 \mathrm{~d}$ ), or preterm at $125-126 \mathrm{~d}$ gestation $(85 \%$ of term gestation) were used in this study.

Ewes were sedated with i.v. pentobarbital sodium (2-4 g total dose) and anesthetized with intrathecal tetracaine hydrochloride ( $1 \%$ solution, $3 \mathrm{mg}$ ). Ewes were kept sedated with pentobarbital sodium but breathed spontaneously throughout the surgery. Under sterile conditions, a uterine incision was made and the fetal head was exteriorized. After local infiltration with lidocaine, a skin incision was made to expose the trachea, internal jugular vein, and carotid artery. Polyvinyl catheters (20G; Martech Medical Products, Lansdale, PA, U.S.A.) were advanced into the ascending aorta through the carotid artery and into the superior vena cava through the jugular vein. The aortic and jugular catheters were connected to Gould-Statham P23 ID pressure transducers (Gould, Inc., Oxnard, CA, U.S.A.) for pressure measurements. Calibrations of pressure were performed with a mercury column manometer. Pancuronium $(0.1 \mathrm{mg} / \mathrm{kg})$ was infused into the fetus, and a tracheotomy was performed with placement of a $3.5-\mathrm{mm}$ internal diameter (ID) (preterm) or 4.0-mm ID (term) endotracheal tube (HiLo Jet Tube, Mallinckrodt Inc., Glenn Falls, NY, U.S.A.). Each animal received an initial infusion of fluid ( $\mathrm{NaCl}$ $0.9 \% ; 10 \mathrm{~mL} / \mathrm{kg}$ ) followed by a continuous infusion of $5 \%$ dextrose in normal saline and pentobarbital $\left(0.5 \mathrm{mg} \cdot \mathrm{kg}^{-1} \cdot \mathrm{h}^{-1}\right)$ at $10 \mathrm{~mL} / \mathrm{h}$.

Mechanical ventilation was initiated in PRVC mode on a Servo 300 ventilator (Siemens, Solna, Sweden) with $\mathrm{Fio}_{2} 1.0$ 
and a set initial TV of $30 \mathrm{~mL}$ for term lambs and $25 \mathrm{~mL}$ for preterm lambs. The PRVC mode delivers the set TV while minimizing pressure within the limits of ventilatory rate and inspiratory time, detecting compliance changes in the subject. Ventilator rate was set at $30 \mathrm{bpm}$ in term animals and $40 \mathrm{bpm}$ in preterm animals. The inspiratory period was $0.75 \mathrm{~s}$, and the PEEP was set to $6 \mathrm{~cm} \mathrm{H}_{2} \mathrm{O}$. Peak inspiratory pressure and $\mathrm{P}_{\text {aw }}$ were recorded from the ventilator. Ventilator rate, inspiratory time, and $\mathrm{FiO}_{2}$ were kept constant during the entire study. After instrumentation, animals were maintained on a radiant warmer. The RIP bands were placed around the chest and abdomen and secured. The bands were calibrated during $\mathrm{CV}$ for all protocols. Animals were killed after the study with high-dose pentobarbital.

\section{Study Design}

The study was divided into the four protocols described below.

Protocol 1: Calibration of the RespiTrace with injected gas volumes. The PV curves were performed as follows: after QDC calibration was completed, the tracheotomy tube was disconnected from the ventilator to EELV with 0 PEEP. $P_{\text {aw }}$ was monitored using the pressure port of the HiLo jet tube attached to a Bird airway pressure monitor (Bird Products Corp., Palm Springs, CA, U.S.A.). Increments of 20-mL aliquots of air (final volume, $120 \mathrm{~mL}$ ) were instilled using a syringe in term animals. After each aliquot was instilled, the $\mathrm{V}_{\mathrm{RIP}}$ value was recorded as well as the airway pressure at the given volume. The deflation limb was characterized in a similar fashion as $20-\mathrm{mL}$ aliquots of air were withdrawn into the syringe. The correlation between $\mathrm{V}_{\text {cal }}$ and $\mathrm{V}_{\mathrm{RIP}}$ was studied and presented as $\mathrm{V} / \mathrm{V}$ curves. This correlation was also shown with PV curves from $\mathrm{V}_{\text {cal }}$ and the $\mathrm{V}_{\mathrm{RIP}}$ at the corresponding airway pressure. All volume measurements were presented as volume changes above EELV, which was set to 0 . A similar protocol was performed in preterm animals except that $15-\mathrm{mL}$ aliquots were used to a maximal volume of $60 \mathrm{~mL}$.

Protocol 2: Changes in lung volume and oxygenation owing to changes in $P_{a w}$ during HFOV in term animals. The relation between changes in $\mathrm{P}_{\text {aw }}$ and $\mathrm{V}_{\mathrm{RIP}}$ was determined during HFOV. Baseline measurements of $V_{\text {RIP }}$ were determined after disconnection from the Siemens ventilator before starting HFOV. HFOV (SensorMedics 3100, SensorMedics Inc., Yorba Linda, CA, U.S.A.) was initiated with a $\mathrm{P}_{\mathrm{aw}}$ of 5 $\mathrm{cm} \mathrm{H}_{2} \mathrm{O}$, which was then increased by $5 \mathrm{~cm}$ of $\mathrm{H}_{2} \mathrm{O}$ at 5 -min intervals to a maximum of $35 \mathrm{~cm} \mathrm{H}_{2} \mathrm{O}$. $\mathrm{P}_{\mathrm{aw}}$ was the only ventilator parameter changed in this study. $\mathrm{V}_{\mathrm{RIP}}$, airway pressures, arterial blood gas values, and BP were recorded at each $\mathrm{P}_{\mathrm{aw}}$ level.

Protocol 3: Changes in lung volume and oxygenation owing to changes in PEEP during CV in term animals. We performed PEEP studies during PRVC mechanical ventilation to compare RIP volume changes with changes in oxygenation. PEEP was the only variable changed on the ventilator during this protocol. PEEP was changed at 5-min intervals in increments of $5 \mathrm{~cm} \mathrm{H}_{2} \mathrm{O}$ to a maximum of $20 \mathrm{~cm} \mathrm{H}_{2} \mathrm{O}$. $\mathrm{V}_{\mathrm{RIP}}$, airway pressures, arterial blood gas values, and BP were recorded at each PEEP level.

Protocol 4: Changes in lung volume owing to changes in elastic properties of the lung during $C V$ in preterm animals before and after surfactant instillation. After QDC calibration, the preterm lambs (125 d) were kept on baseline settings of mechanical ventilation as described above (PRVC mode set with a TV of $25 \mathrm{~mL}$, ventilator rate at $40 \mathrm{bpm}$, inspiratory period of $0.75 \mathrm{~s}$, and a PEEP of $6 \mathrm{~cm} \mathrm{H}_{2} \mathrm{O}$; resulting peak inspiratory pressure and $\mathrm{P}_{\mathrm{aw}}$ were recorded from the ventilator). In three animals, we compared PV curves before and after surfactant instillation to assess whether RIP could detect an acute change in compliance and lung volume. A baseline PV curve before surfactant was performed after the manifestations of RDS became severe $\left[\mathrm{PaO}_{2}<13.3 \mathrm{kPa}\right.$ (100 mm Hg) despite ventilation with $\mathrm{FiO}_{2}$ 1.0]. Surfactant (Infasurf, provided by E.A. Egan, M.D., State University of New York at Buffalo) was then infused over 2-5 min through a side port of the tracheotomy tube during baseline ventilation. A new PV curve was done $30 \mathrm{~min}$ after surfactant instillation.

\section{Study Measurements}

The value of the electrical inductance obtained from the computer connected to the RIP monitor in this study was called $\mathrm{V}_{\mathrm{RIP}} \cdot \mathrm{V}_{\mathrm{RIP}}$ was expressed as the $\Delta$ change in the figures to allow grouping of the animals. Ventilator settings and pressures were registered from the ventilators (Servo 300 and SensorMedics 3100). An arterial blood sample was obtained after each change in ventilator settings for measurements of $\mathrm{pH}, \mathrm{PaO}_{2}$, and $\mathrm{PaCO}_{2}$ (Radiometer OSM 3; Copenhagen, Denmark). BP and heart rate were measured throughout the study.

\section{Data Analysis}

The injected gas volume per kilogram body weight was plotted against $\mathrm{V}_{\mathrm{RIP}}$ for term and preterm animals. The relationship between these values during lung inflation and deflation was assessed by linear regression analysis, providing $r^{2}$ values. The raw data were presented as V/V curves. PV curves were plotted from $\mathrm{V}_{\text {cal }}$ and $\mathrm{V}_{\text {RIP }}$ against the $\mathrm{P}_{\mathrm{aw}}$. The EELV when the animals were disconnected from the ventilator was set as the zero point. Data were also plotted as PV curves from term and preterm animals as well as data on preterm animals before and after surfactant instillation. Dynamic changes in $\mathrm{V}_{\mathrm{RIP}}$ in response to changes in $\mathrm{P}_{\mathrm{aw}}$ on HFOV were plotted over time along with concomitant changes in $\mathrm{PaO}_{2} \mathrm{~A}$ similar plot was made with $\mathrm{V}_{\text {RIP }}$ and changes in PEEP and $\mathrm{PaO}_{2}$ on PRVC. Linear regression analysis was performed using the computer program StatMost 3.5 (Dataxiom Software Inc., Los Angeles, CA, U.S.A.). Descriptive statistics and graphs were performed with Axum 5.0 (MathSoft International, Bogshot, Surrey, UK).

\section{RESULTS}

Fourteen lambs were studied in two groups, six near-term animals (mean gestational age, $140 \pm 3 \mathrm{~d}$; term $=147 \mathrm{~d}$; mean birth weight, $3370 \pm 290 \mathrm{~g}$ ) and eight preterm lambs (at $125 \mathrm{~d}$ gestation; mean birth weight, $2410 \pm 250 \mathrm{~g}$ ). 
Figure 1 shows a trend tracing computed from the RIP signal during a pilot recruitment/derecruitment procedure during HFOV in one animal. It shows the hysteresis of the lungs with an increased lung volume at similar $\mathrm{P}_{\mathrm{aw}}$ after recruitment.

Protocol 1: Calibration of the RespiTrace with injected gas volumes. Linear regression analysis of the inflation limbs between injected gas volume and the lung volume in the term lambs yielded $r^{2}$ values between $0.98-0.99$ (Fig. 2a). The $r^{2}$ values for the deflation limbs were $0.93-1.00$ (Fig. $2 b$ ). The $r^{2}$ values of the linear regression in the preterm lambs for the inflation limbs were $0.98-1.00$ (Fig. 2c) and for the deflation limbs, 0.86-1.00 (Fig. 2d).

The PV curves for both term and preterm lambs with mean values of the $\mathrm{P}_{\mathrm{aw}} / \mathrm{V}_{\mathrm{cal}}$ and $\mathrm{P}_{\mathrm{aw}} / \mathrm{V}_{\mathrm{RIP}}$ for a given vol $/ \mathrm{kg} \mathrm{dem}$ onstrate marked differences in hysteresis between the term (Fig. 3a) and preterm lambs (Fig. 3b).

Protocol 2: Changes in lung volume and oxygenation owing to changes in $P_{a w}$ during $\mathrm{HFOV}$ in term animals. Mean changes in oxygenation and $\mathrm{V}_{\text {RIP }}$ during recruitment and derecruitment maneuvers during changes in $\mathrm{P}_{\mathrm{aw}}$ in term lambs on HFOV are plotted in Figure 4, $a$ and $b . \mathrm{PaO}_{2}$ increased with each change in $\mathrm{P}_{\text {aw }}$ up to $25 \mathrm{~cm} \mathrm{H}_{2} \mathrm{O}$ (Fig. 4a). As $\mathrm{P}_{\text {aw }}$ was further increased to $35 \mathrm{~cm} \mathrm{H}_{2} \mathrm{O}, \mathrm{V}_{\mathrm{RIP}}$ did not increase further, indicating that the lung had been inflated onto the asymptote of the PV curve, and $\mathrm{PaO}_{2}$ fell concomitantly. As $\mathrm{P}_{\text {aw }}$ was reduced from 35 to $10 \mathrm{~cm} \mathrm{H} \mathrm{H}_{2} \mathrm{O}, \mathrm{PaO}_{2}$ increased (Fig. 4b). Marked reduction in thoracic gas volume occurred at a $\mathrm{P}_{\mathrm{aw}}$ of $5 \mathrm{~cm}$ $\mathrm{H}_{2} \mathrm{O}$, reflected as a fall in $\mathrm{V}_{\mathrm{RIP}}$ and $\mathrm{PaO}_{2}$. The loss of lung volume was detected by RIP before the deterioration in oxygenation started.

Protocol 3: Changes in lung volume and oxygenation owing to changes in PEEP during $C V$ in term animals. Changes in oxygenation and $\mathrm{V}_{\text {RIP }}$ during recruitment and derecruitment maneuvers with alterations in PEEP in term lambs during PRVC are shown in Figure 5, $a$ and $b . \mathrm{PaO}_{2}$ increased with each change in PEEP up to $10 \mathrm{~cm} \mathrm{H}_{2} \mathrm{O}$ (Fig. 5a). As PEEP was increased from 10 to $20 \mathrm{~cm} \mathrm{H}_{2} \mathrm{O}, \mathrm{PaO}_{2}$ progressively fell, possibly reflecting hyperinflation. $\mathrm{PaO}_{2}$ increased as PEEP was reduced from 20 to $5 \mathrm{~cm} \mathrm{H}_{2} \mathrm{O}$ (Fig. 5b). Marked reduction in lung volume occurred at a PEEP of 0 , reflected as a fall in $\mathrm{V}_{\mathrm{RIP}}$ and $\mathrm{PaO}_{2}$. The loss of lung volume was detected by RIP before the deterioration in oxygenation started.

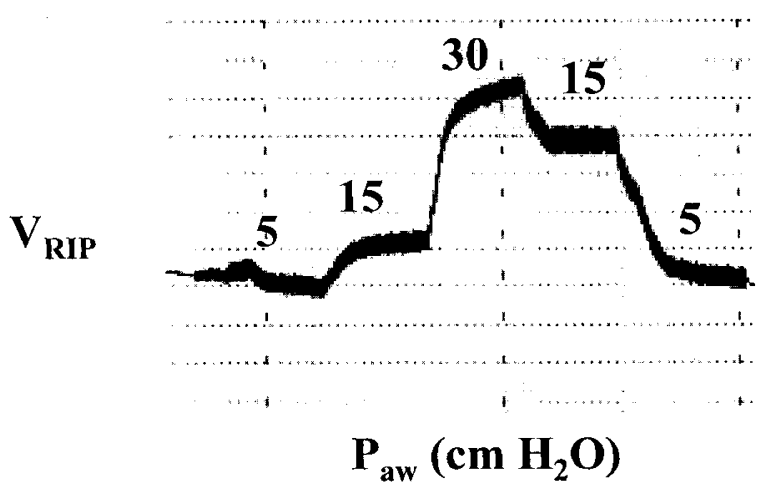

Figure 1. Sample tracing from RIP showing the change in mean thoracic gas volume $\left(\mathrm{V}_{\mathrm{RIP}}\right)$ for changes in $\mathrm{P}_{\mathrm{aw}}$ in one animal on HFOV.
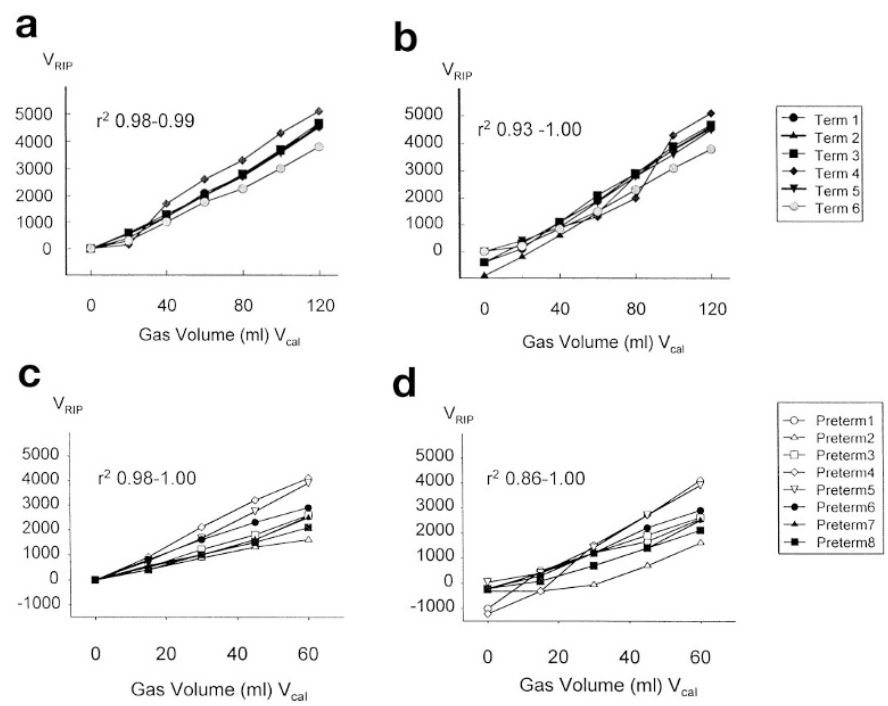

Figure 2. Regression curves comparing the change in the thoracic gas volume $\left(\mathrm{V}_{\mathrm{RIP}}\right)$ as registered by RIP with $\mathrm{V}_{\text {cal }}$ during $(a)$ inflation limb/term, $(b)$ deflation limb/term, $(c)$ inflation limb/preterm, and $(d)$ deflation limb/preterm.
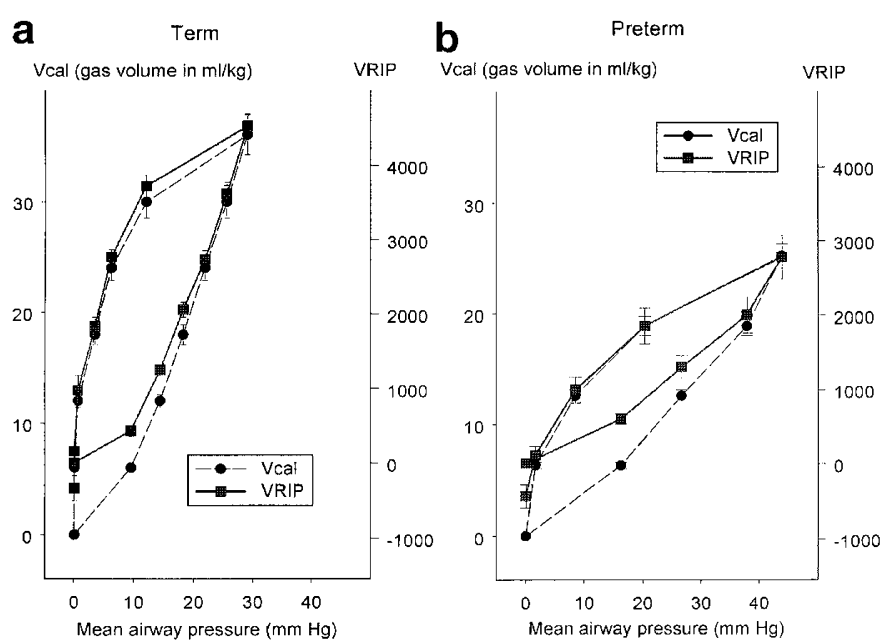

Figure 3. PV curves from term animals $(n=6)(a)$ and preterm animals $(n=$ 8) $(b)$ with the injected gas volume $(\mathrm{mL} / \mathrm{kg}) \pm \mathrm{SEM}$ on the left $y$ axis (-O$\mathrm{V}_{\text {cal }}$ ) and the mean $\mathrm{V}_{\mathrm{RIP}} \pm \mathrm{SEM}$ from the RespiTrace on the right $y$ axis $\left(-\|-\mathrm{V}_{\mathrm{RIP}}\right) \cdot x$ axis expresses the $\mathrm{P}_{\mathrm{aw}}\left(\mathrm{cm} \mathrm{H}_{2} \mathrm{O}\right)$ as read from the pressure monitor.

Protocol 4: Changes in lung volume owing to changes in elastic properties of the lung during $C V$ in preterm animals before and after surfactant instillation. The mean values of $\mathrm{V}_{\text {RIP }}$ and $\mathrm{P}_{\mathrm{aw}}$ in preterm lambs before and after surfactant treatment are plotted as PV curves (Fig. 6). Surfactant-treated lungs had larger volumes for the same airway pressures during inflation and deflation.

\section{DISCUSSION}

In this study, we used RIP to measure relative lung volume $\left(\mathrm{V}_{\mathrm{RIP}}\right)$ changes during HFOV, during $\mathrm{CV}$, and after surfactant replacement in premature animals. Linear regression analysis comparing $\mathrm{V}_{\mathrm{RIP}}$ with injections of $\mathrm{V}_{\text {cal }}$ showed good correlation. $\mathrm{V}_{\mathrm{RIP}}$ also correlated well to changes in $\mathrm{P}_{\mathrm{aw}}$ on HFOV. As 

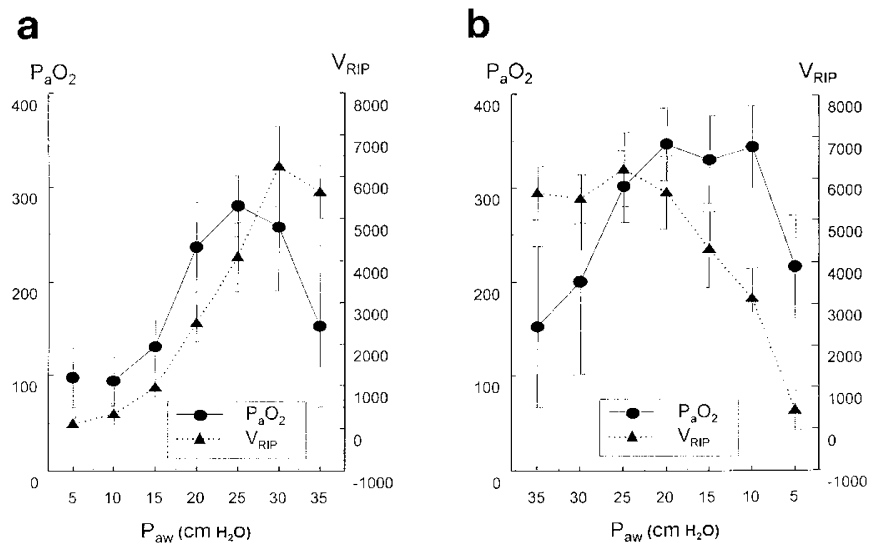

Figure 4. Parallel changes in mean $\mathrm{PaO}_{2} \pm \mathrm{SEM}(-\bullet-)$ and mean $\mathrm{V}_{\mathrm{RIP}} \pm \mathrm{SEM}$ (- $\mathbf{A}-)$ in term animals on HFOV during $(a)$ recruitment and $(b)$ derecruitment.
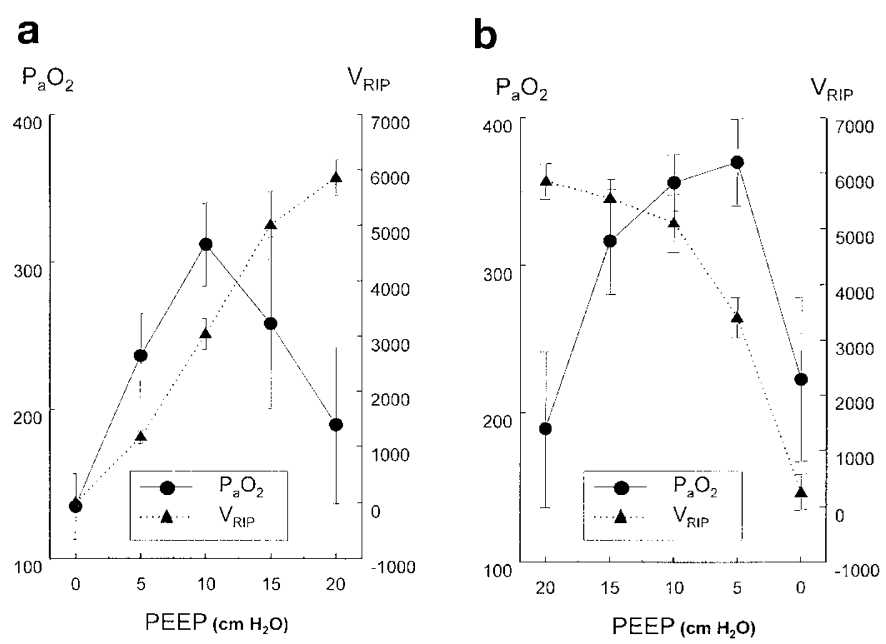

Figure 5. Parallel changes in mean $\mathrm{PaO}_{2} \pm \mathrm{SEM}(-\boldsymbol{\bullet}-)$ and mean $\mathrm{V}_{\mathrm{RIP}} \pm \mathrm{SEM}$ (- - -) in term animals on PRVC mechanical ventilation during $(a)$ recruitment and $(b)$ derecruitment.

$\mathrm{P}_{\mathrm{aw}}$ was decreased, a decrease in lung volume was detected by RIP before severe deterioration in oxygenation occurred.

These findings are important, as HFOV is increasingly used in the management of neonatal and pediatric respiratory failure because of its efficacy in lung volume recruitment without tidal ventilation and without TV-induced lung injury. The use of HFOV with a strategy designed to recruit and sustain adequate lung volume has improved oxygenation in newborns with hypoxemic respiratory failure $(40,41)$. Lack of success when instituting HFOV is often related to failure in reaching an adequate $\mathrm{P}_{\mathrm{aw}}$ to cause lung recruitment. However, adverse effects from lung hyperinflation may occur if $\mathrm{P}_{\mathrm{aw}}$ is not appropriately reduced after lung volume and compliance have improved (19). One previously published study used the combination of an early RIP device and HFOV to detect air trapping during combined HFOV and intermittent mandatory ventilation treatment (42). Monitoring lung volume during HFOV is clinically difficult but remains critical for the successful application of this therapy (12). Of equal importance, measurements of lung volume during HFOV and $\mathrm{CV}$ after lung recruitment maneuvers will allow for reduction of $\mathrm{P}_{\mathrm{aw}}$ without loss of lung

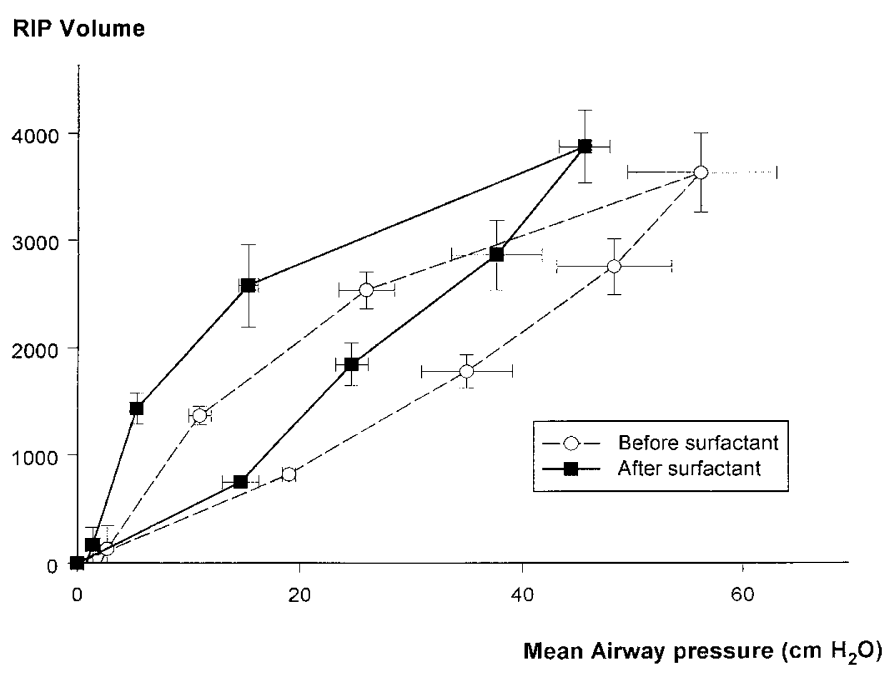

Figure 6. PV curves from preterm animals before (-0-) and after (- $-(-)$ surfactant instillation $(n=3)$. The $y$ axis represents the mean $\mathrm{V}_{\mathrm{RIP}} \pm \mathrm{SEM}$, and the $x$ axis represents the $\mathrm{P}_{\mathrm{aw}}\left(\mathrm{cm} \mathrm{H}_{2} \mathrm{O}\right) \pm$ SEM as read from the pressure monitor. Note the left and upward shifting in PV curve and the increased hysteresis after surfactant.

volume by derecruitment (13). Standard methods for static volume measurements, pneumotachography, and indicator gas washout techniques are not applicable to HFOV. This study demonstrates that the application of RIP technology can enhance assessments of lung volume during HFOV.

We also found that dynamic changes in $V_{R I P}$ paralleled changes in PEEP during PRVC ventilation. Although there are several ways to measure static lung volumes on $\mathrm{CV}$, there are some limitations when measuring rapid dynamic responses, and bedside use is cumbersome. The reduction of $\mathrm{P}_{\mathrm{aw}}$ needed to sustain the same lung volume after surfactant treatment was clearly demonstrated using this technique. In contrast with a previous study (27), we found that there was rapid stabilization of the $V_{\text {RIP }}$ signal (within three to five breaths).

Considering the vital role of optimizing lung volume in the management of hypoxemic respiratory failure, the RIP technique has the potential for directly targeting lung volumes and ventilating on the deflation limb of the PV curve during mechanical ventilation. Potential applications of this technique during HFOV include verifying that an effective volume recruitment pressure $\left(\mathrm{P}_{\mathrm{aw}}\right)$ is applied after converting from $\mathrm{CV}$ to HFOV. Other potential areas of use are restoring reduced lung volume after disconnection of the ventilatory circuit and providing lung volume measurements when weaning from ventilator support. There are also important implications of RIP measurements when adjusting ventilator settings during CV. Ventilation-induced lung injury is a significant complication of TV ventilation, associated with cyclic stretch stress that precipitates alveolar leak and inflammatory lung injury (26). The technique described in this article may provide critical information about incipient lung overinflation. However, of equal importance is the potential to reduce lung injury caused by insufficient lung recruitment and low functional residual capacity (20). Thus, a lung volume-targeted strategy may offer advantages over the traditional focus on gas exchange alone, 
and the technique described here may provide such information in a noninvasive fashion.

Early studies in which older variations of RIP were used have dealt with static lung volume measurements, and they have found good correlation for TV measurements when compared with pneumotachography (31-33, 43-45). However, recent studies in adults have yielded somewhat conflicting results $(46,47)$. We found good correlation between $V_{\text {RIP }}$ and injected gas volumes, perhaps due to the highly compliant chest wall of the newborn. It is possible that lung volume changes are more easily detected by the thoracic bands of the RIP system in the newborn compared with the adult. Edberg et al. (8) have shown an increased resting volume after surfactant instillation in preterm babies that could not be seen in our study. This difference is probably due to differences in the technique of establishing the resting volume. In the study by Edberg et al. (8), this volume was measured by means of a nitrogen washout technique in a face-out whole body plethysmograph without disconnection of the tracheal tube from the ventilator, whereas, in our study, the EELV was established through disconnection of the tube from the ventilator. This may explain why we did not see an increase in EELV after surfactant, as we may have lost the gained resting volume whereas they did not. The increase in lung compliance is nevertheless clearly shown with the RespiTrace, and a much lower $\mathrm{P}_{\mathrm{aw}}$ was needed to recruit the lungs after surfactant.

There are several limitations of this technique. Changes in thoracic and/or abdominal band position may change the baseline $\mathrm{V}_{\mathrm{RIP}}$ recording. Also, the RIP system works with a Wheatstone bridge and bands, which are sensitive to changes in ambient temperature. Environmental temperature fluctuations may affect the resistance of the bridge, which could influence recorded changes in lung volume even in the DC-coupled mode. In the system we tested, the oscillatory module has been moved into the electronic enclosure to protect it from the changes in environmental temperature. However, the effect of ambient temperature on the thoracic and abdominal bands must be considered when monitoring infants nursed under radiant warmers. It is also important to stress that the changes in lung volume inferred from the changes in electrical inductance with RIP are relative and not absolute.

We conclude that acute measurements of lung volume by use of modified RIP technology parallel well changes in $\mathrm{P}_{\text {aw }}$ during HFOV and also with rapid dynamic changes during $\mathrm{CV}$. We speculate that this technique may provide clinically useful information about acute changes in lung volume, especially during nonconventional non-TV mechanical ventilation. However, determinations of precision and chronic stability of the technique over longer duration require further studies.

Acknowledgments. The RIP system with the new software was kindly provided by Alex Stenzler, SensorMedics, Inc., Yorba Linda, CA, U.S.A. The Servo 300 ventilator was kindly provided by Christer Ström, Siemens, Solna, Sweden.

\section{REFERENCES}

1. Greenfield LJ, Ebert PA, Benson DW 1964 Effect of positive pressure ventilation on surface tension properties of lung extracts. Anesthesiology 25:312-316
2. Kolobow T, Moretti MP, Fumagalli R, Mascheroni D, Prato P, Chen V, Joris M 1987 Severe impairment in lung function induced by high peak airway pressure during mechanical ventilation. Am Rev Respir Dis 135:312-315

3. Walsh MC, Carlo WA 1988 Sustained inflation during HFOV improves pulmonary mechanics and oxygenation. J Appl Physiol 65:368-372

4. Kinsella JP, Gerstmann DR, Clark RH, Null DM, Morrow WR, Taylor AF, de Lemos RA 1991 High-frequency oscillatory ventilation versus intermittent mandatory ventilation: early hemodynamic effects in the premature baboon with hyaline membrane disease. Pediatr Res 29:160-166

5. Richardson P, Bose CL, Carlstroem JR 1986 The functional residual capacity of infants with respiratory distress syndrome. Acta Paediatr Scand 75:267-271

6. Edberg KE, Sandberg K, Silberberg A, Ekstrom-Jodal B, Hjalmarson O 1991 Lung volume, gas mixing, and mechanics of breathing in mechanically ventilated very low birth weight infants with idiopathic respiratory distress syndrome. Pediatr Res 30:496-500

7. Sandberg K, Edberg KE, Benton W, Silberberg A, Sladek M, Sundell HW 1991 Surfactant improves gas mixing and alveolar ventilation in preterm lambs. Pediatr Res 30:181-189

8. Edberg KE, Ekstrom-Jodal B, Hallman M, Hjalmarsson O, Sandberg K, Silberberg A 1990 Immediate effects on lung function of instilled human surfactant in mechanically ventilated newborn infants with IRDS. Acta Paediatr Scand 79:750-755

9. Kinsella JP, Abman SH 1996 Clinical approaches to the use of high-frequency oscillatory ventilation in neonatal respiratory failure. J Perinatol 16:S52-S55

10. Matamis D, Lemaire F, Harf A, Brun-Buisson C, Ansquer JC, Atlan G 1984 Total respiratory pressure-volume curves in the adult respiratory distress syndrome. Chest $86: 58-66$

11. Gattinoni L, Pesenti A, Avalli L, Rossi F, Bombino M 1987 Pressure-volume curve of total respiratory system in acute respiratory failure. Computed tomographic scan study. Am Rev Respir Dis 136:730-736

12. Bryan AC, Slutsky AS 1986 Lung volume during high-frequency oscillation. Am Rey Respir Dis 133:928-930

13. Rimensberger PC, Cox PN, Frndova H, Bryan AC 1999 The open lung during small tidal volume ventilation: concepts of recruitment and "optimal" positive endexpiratory pressure. Crit Care Med 27:1946-1952

14. Alexander J, Milner AD 1997 Determination of gas-trapping during high-frequency oscillatory ventilation. Acta Paediatr 86:268-273

15. Amato MBP, Barbas CSV, Medeiros DM, de Paula Pinto Schettino G, Filho GL, Kairalla RA, Deheinzelin D, Morais C, de Oliveira Fernandes E, Yakagaki TY, de Carvalho CRR 1995 Beneficial effects of the "open lung approach" with low distending pressures in acute respiratory distress syndrome. Am J Respir Crit Care Med 152:1835-1846

16. Byford LJ, Finkler JH, Froese AB 1988 Lung volume recruitment during highfrequency oscillation in atelectasis-prone rabbits. J Appl Physiol 64:1607-1614

17. Vasquez de Anda GF, Hartog A, Verbrugge SJ, Gommers D, Lachmann B 1999 The open lung concept: pressure-controlled ventilation is as effective as high-frequency oscillatory ventilation in improving gas exchange and lung mechanics in surfactantdeficient animals. Intensive Care Med 25:990-996

18. Webb HH, Tierney DF 1974 Experimental pulmonary edema due to intermittent positive pressure ventilation with high inflation pressures. Protection by positive end-expiratory pressure. Am Rev Respir Dis 110:556-565

19. McCulloch PR, Forket PG, Froese AB 1988 Lung volume maintenance prevents lung injury during high-frequency oscillatory ventilation in surfactant-deficient rabbits. Am Rev Respir Dis 137:1185-1192

20. Muscedere JG, Mullen JB, Slutsky AS 1994 Tidal ventilation at low airway pressures can augment lung injury. Am J Respir Crit Care Med 149:1327-1334

21. Clark RH, Gerstmann DR, Null DMJ, de Lemos RA 1992 Prospective randomized comparison of high-frequency oscillatory and conventional ventilation in respiratory distress syndrome. Pediatrics 89:5-12

22. de Lemos RA, Coalson JJ, Gerstmann DR, Null DMJ, Ackerman NB, Escobedo MB, Robotham JL, Kuehl TJ 1987 Ventilatory management of infant baboons with hyaline membrane disease: the use of high-frequency ventilation. Pediatr Res 21:594-602

23. Dreyfuss D, Soler P, Basset G, Saumon G 1988 High inflation pressure pulmonary edema. Respective effects of high airway pressure, high tidal volume, and positive end-expiratory pressure. Am Rev Respir Dis 137:1159-1164

24. Kinsella JP, Parker TA, Galan H, Sheridan BC, Abman SH 1999 Independent and combined effects of inhaled nitric oxide, liquid perfluorochemical and high-frequency oscillatory ventilation in premature lambs with respiratory distress syndrome. Am J Respir Crit Care Med 159:1220-1227

25. Jobe AH, Ikegami M 1998 Mechanisms initiating lung injury in the preterm. Early Hum Dev 53:81-94

26. Dreyfuss D, Saumon G 1998 Ventilator-induced lung injury. Am J Respir Crit Care Med 157:294-323

27. Thome U, Töpfer A, Schaller P, Pohlandt F 1998 Effect of mean airway pressure on lung volume during high-frequency oscillatory ventilation of preterm infants. Am J Respir Crit Care Med 157:1213-1218

28. Imanaka H, Takezawa J, Nishimura M, Nishijima M, Taenaka N, Yoshija I 1990 Measurement of functional residual capacity during high-frequency oscillatory ventilation (HFOV) by argon washout method without interruption of HFOV. Chest 97:1152-1156

29. Watson H 1980 The technology of respiratory inductive plethysmography. Proceedings of the Third International Symposium in Ambulatory Monitoring, London, pp 537-563

30. Cohn MA, Watson H, Weisshaut R, Stott F, Sackner MA 1978 A transducer for noninvasive monitoring of respiration. Proceedings of the Second International Symposium in Ambulatory Monitoring, London, pp 119-128 
31. Duffty P, Spriet L, Bryan MH, Bryan AC 1981 Respiratory induction plethysmography (RespiTrace): an evaluation of its use in the infant. Am Rev Respir Dis 123:542-546

32. Tabachnik E, Muller N, Toye B, Levison H 1981 Measurement of ventilation in children using the respiratory inductive plethysmograph. J Pediatr 99:895-899

33. Dolfin T, Duffty P, Wilkes DL, Bryan MH 1982 Calibration of respiratory induction plethysmography (RespiTrace) in infants. Am Rev Respir Dis 126:577-579

34. Stefano JL, Spitzer AR, Baumgart S, Davis JM, Fox WW 1986 Inductive plethysmography-a facilitated postural calibration technique for rapid and accurate tida volume determination in low birth weight premature newborns. Am Rev Respir Dis 134:1020-1024

35. Strömberg NOT, Dahlbäck GO, Gustafsson PM 1993 Evaluation of various models for respiratory inductance plethysmography calibration. J Appl Physiol 74:12061211

36. Jobe AH 1992 Surfactant in the perinatal period. Early Hum Dev 29:57-62

37. Walther FJ, Benders MJ, Leighton JO 1992 Persistent pulmonary hypertension in premature neonates with severe respiratory distress syndrome. Pediatrics 90:899-904

38. Konno K, Mead J 1967 Measurement of the separate volume changes of rib cage and abdomen during breathing. J Appl Physiol 22:407-422

39. Sackner MA, Watson H, Belsito AS, Feinerman D, Suarez M, Gonzalez G, Bizousky F, Krieger B 1989 Calibration of respiratory inductive plethysmography during natural breathing. J Appl Physiol 66:410-420

40. Clark RH 1994 High-frequency ventilation. J Pediatr 124:661-670
41. Kinsella JP, Troug WE, Walsh WF, Goldberg RN, Bancalari E, Mayock DE, Redding JJ, de Lemos RA, Sardesai S, McCurnin DCea 1997 Randomized multicenter trial of inhaled nitric oxide and high-frequency oscillatory ventilation in severe, persistent pulmonary hypertension of the newborn. J Pediatr 131:55-62

42. Smith DW, Frankel LR, Ariagno RL 1988 Dissociation of mean airway pressure and lung volume during high-frequency oscillatory ventilation. Crit Care Med 16:531535

43. Tobin MJ, Jenouri G, Lind B, Watson H, Schneider A, Sackner MA 1983 Validation of respiratory inductive plethysmography in patients with pulmonary disease. Chest 83:615-620

44. Revow MD, England SJ, Stogryn HAF, Wilkes DL 1987 Comparison of calibration methods for respiratory inductive plethysmography in infants. J Appl Physiol 63:1853-1861

45. Carry PY, Baconnier P, Eberhard A, Cotte P, Benchetrit G 1997 Evaluation of respiratory inductive plethysmography. Accuracy for analysis of waveforms. Chest 111:910-915

46. Valta P, Takala J, Foster R, Weissman C, Kinney JM 1992 Evaluation of respiratory inductive plethysmography in the measurement of breathing pattern and PEEPinduced changes in lung volume. Chest 102:234-238

47. Neumann P, Zinserling J, Haase C, Sydow M, Burchardi H 1998 Evaluation of respiratory inductive plethysmography in controlled ventilation. Measurement of tidal volume and PEEP-induced changes of end-expiratory lung volume. Chest 113:443451 